\title{
Le nouveau paradigme de la violence (Partie 2)
}

\author{
Michel Wieviorka
}

\section{OpenEdition}

\section{Journals}

\section{Édition électronique}

URL : http://journals.openedition.org/conflits/726

DOI : $10.4000 /$ conflits.726

ISSN : $1777-5345$

Éditeur :

CCLS - Centre d'études sur les conflits lilberté et sécurité, L'Harmattan

\section{Édition imprimée}

Date de publication : 15 octobre 1998

ISSN : 1157-996X

Référence électronique

Michel Wieviorka, «Le nouveau paradigme de la violence (Partie 2) », Cultures \& Conflits [En ligne], 29-30 | automne-hiver 1998, mis en ligne le 16 mars 2006, consulté le 30 mars 2021. URL : http:// journals.openedition.org/conflits/726 ; DOI : https://doi.org/10.4000/conflits.726

Ce document a été généré automatiquement le 30 mars 2021.

Creative Commons License 


\title{
Le nouveau paradigme de la violence (Partie 2)
}

\author{
Michel Wieviorka
}

1 Quatre niveaux d'analyse

2 L'idée d'un nouveau paradigme est donc confortée par l'examen des changements qui renvoient aux significations, aux perceptions et aux modes d'approche de la violence. Elle n'est pas pour autant totalement établie ou démontrée, ne serait-ce qu'en raison des inflexions et des renversements de tendance qui peuvent toujours survenir dans une évolution historique. C'est pourquoi elle appelle des investigations complémentaires, à commencer par celles qui peuvent porter sur les changements relatifs aux principales sources de la violence depuis les années soixante-dix.

3 Il est classique, dans l'analyse de la violence, de distinguer des niveaux. Dans les années soixante, par exemple, Pierre Hassner demandait que l'on en différencie trois. Le premier était celui du système international, dont il dit qu'il renvoyait alors " à l'équilibre bipolaire de la dissuasion et, en Europe, à la division territoriale des deux blocs " ${ }^{1}$; le deuxième était celui des Etats, avec leurs préoccupations intérieures et diplomatiques, et le troisième celui des sociétés, à l'intérieur des Etats, avec chacune son système politique, ses structures et leur dynamique. Cette distinction, que nous avons déjà utilisée dans des travaux portant sur le terrorisme des années soixante-dix et quatre-vingt ${ }^{2}$, permet de réfléchir aux conditions générales du changement de paradigme de la violence, et nous la reprendrons ici, en ajoutant simplement un quatrième niveau, celui de l'individu, non pas pour introduire une quelconque psychologie dans nos analyses, mais pour insister sur un phénomène contemporain majeur, et qui pèse lourdement sur la production de la violence contemporaine : la poussée de l'individualisme moderne. A chacun de ces quatre niveaux, les changements récents sont considérables, et en les envisageant, on apporte déjà un éclairage utile sur les phénomènes de violence. Cet éclairage est complété et précisé si l'analyse prend en compte, de surcroît, les transformations qui affectent les relations entre les niveaux, leurs articulations, leur correspondance, ou, si l'on préfère, leur intégration.

Le système international 

l'Empire soviétique et le dépérissement de la bipolarité Est-Ouest. Avec cette transformation décisive, la division de l'Europe est elle-même liquidée, ou plutôt appelle d'autres catégories pour être pensée : n'y a-t-il pas, par exemple, les pays de l'ancien Empire, principalement la Pologne, la Hongrie et la République tchèque, qui basculent à l'ouest politiquement (la démocratie) et économiquement (le marché), tandis que les autres hésitent à se dégager des anciens modèles communistes, ou n'y parviennent que très partiellement? La fin de la guerre froide, c'est aussi l'entrée dans une nouvelle ère du point de vue des armes nucléaires. La dissuasion nucléaire ne peut plus être pensée aujourd'hui comme elle l'était hier, elle n'apporte plus la raison dans un univers bipolaire structurant l'essentiel des relations interétatiques à partir des deux superpuissances, et le nucléaire devient le symbole de risques majeurs de crise, de déstabilisation, de terrorisme éventuellement, sans parler de la simple catastrophe que peuvent causer un jour ou l'autre la prolifération des armes nucléaires et la dissémination de matières fissiles depuis l'ex-Union soviétique. Pierre Hassner dit que les armes nucléaires " deviennent l'exemple extrême non de l'ordre mais du fossé entre le caractère global et diffus des problèmes et le caractère partiel et spécialisé des organismes chargés de les gérer ou de les contrôler "3, on est tenté d'ajouter qu'on ne voit pas toujours, avec la désintégration du système soviétique et la fin de la guerre froide, quels sont les organismes capables, même partiellement, d'agir efficacement face à des problèmes comme ceux que pose la dissémination nucléaire.

7 Le nucléaire de la guerre froide rendait peu probable, ou moins probable, la guerre entre de nombreux Etats, il apportait un contrôle partiel et inégal, mais réel, de la violence, un ordre effectivement planétaire, puisque combiné au principe de bipolarité, il interdisait la montée des violences à l'extrême, pour les deux superpuissances, mais aussi partout dans le monde : tout déplacement d'un équilibre, même local, risquait de générer l'escalade de la tension et des déséquilibres majeurs. La planète est sortie de l'ordre nucléaire, pour reprendre l'expression de Philippe Delmas ${ }^{4}$, sans pour autant être entrée dans une ère post-nucléaire. Dès lors, conflits et violences localisés, de basse intensité, ont plus d'espace pour surgir, et il devient difficile d'empêcher qu'ils ne dégénèrent en une barbarie massive dont les massacres du Rwanda ou de l'exYougoslavie ne sont peut-être que les premières expressions. La fin de la guerre froide ne doit rien en elle-même à des violences significatives, et beaucoup à la décomposition et à l'épuisement du régime soviétique ; mais elle a pour conséquence des violences, dont les plus immédiates sont celles qui surgissent au sein même de l'ancien empire soviétique, à commencer par celles du Caucase et de la guerre de la Russie de Boris Eltsine avec la Tchétchénie.

8 Il est possible que la guerre froide ait fonctionné aussi comme facteur d'aveuglement sur les déterminants et les significations locaux de diverses expériences de violence des années cinquante à quatre-vingt, et que ce qui semble nouveau - l'importance des facteurs liés au jeu des acteurs sur le terrain, et non plus celle d'influences extérieures lointaines - soit en réalité surtout plus visible, ou mieux perçu. Mais l'analyse serrée de certaines de ces expériences par des auteurs attentifs à cette hypothèse montre que la fin de la guerre froide a bien apporté des modifications considérables ${ }^{5}$. 
9 Un deuxième changement majeur est celui que l'expression de " globalisation de l'économie " a popularisé partout dans le monde. Le phénomène n'est pas nouveau, et on trouverait chez de nombreux auteurs du XIXe siècle, par exemple chez Karl Marx, des analyses qui renvoient à l'idée d'une mondialisation de l'économie. Ce qui semble décisif, par contre, et même si l'idée en est combattue, est l'accélération du phénomène, que de nombreux économistes datent du milieu des années quatre-vingt. La notion de globalisation signifie que les économies nationales sont de plus en plus interdépendantes, que la croissance de leurs échanges est supérieure à celle de leur production, que les investissements et les flux financiers se mondialisent sous l'effet conjugué de la libéralisation des marchés et des progrès technologiques ${ }^{6}$. La mondialisation de l'économie mérite assurément mieux que des affirmations rapides et de plus en plus stéréotypées, qui font de ce qu'on appelle moins justement la globalisation un " mythe récurrent ", selon l'expression d'Elie Cohen, qui nous invite à éviter les illusions d'une rhétorique incantatoire où cette notion joue un rôle central ${ }^{7}$. Il n'est pas pour autant inutile d'évoquer les liens entre la mondialisation et le néolibéralisme qui la fonde idéologiquement, et la violence. En effet, celle-ci se nourrit, ne serait-ce qu'indirectement, des inégalités et de l'exclusion qui se renforcent avec le marché généralisé, la libre entreprise, la rigueur budgétaire et le libre-échange, et elle est sensible aux évolutions qui rendent l'échange plus important que la production et qui mettent en cause le travail, aussi bien comme signification centrale, comme sens de l'expérience humaine, que comme facteur étroitement associé à la croissance. De plus, la violence peut s'inscrire dans le prolongement de la fragmentation culturelle qu'encourage la mondialisation de l'économie (mais aussi qui l'encourage), selon des processus que Benjamin Barber fut un des premiers à décrire, dès le début des années quatre-vingt-dix ${ }^{8}$. Contrairement à une idée simpliste, la mondialisation en effet, y compris dans ses aspects liés à la diffusion de biens culturels (programmes de télévision par exemple) ne contribue pas seulement à homogénéiser culturellement la planète, elle a aussi, et surtout, pour effet de stimuler des processus plus ou moins réactifs de repli identitaire, du communautarisme, du nationalisme rétracté de façon à défendre la nation contre la culture cosmopolite ou transnationale sous hégémonie nordaméricaine, etc. - processus qui eux-mêmes nourrissent en retour le phénomène. Et de là, il n'est pas difficile de comprendre comment la violence vient traduire éventuellement en actes la volonté défensive, voire contre-offensive, de groupes désireux d'affirmer leur identité culturelle. Paradoxalement, le résultat peut être le même lorsqu'un groupe s'adosse sur une identité de ce type non pour résister à l'économie mondialisée, mais pour mieux y participer.

10 Ces dimensions de la mondialisation peuvent être lues à l'échelle planétaire, comme autant de fractures, sociales et culturelles, qui séparent les pays ou les régions bien placées, " in ", de ceux qui ne le sont pas, " out ". Elles jouent aussi, et très fortement, à l'intérieur des sociétés les plus développées, où se développent aussi bien la misère, l'exclusion, et les formes de discrimination sociale et raciale qui vont généralement de pair, que la fragmentation culturelle, avec ses éventuelles modalités communautaires, tribales ou, là encore, raciales. La mondialisation économique est dans une relation dialectique avec la fragmentation sociale et culturelle qui tout à la fois l'alimente et s'en approfondit, et qui se prolonge par des processus de naturalisation et, plus précisément, de racialisation de la vie collective, avec ce que cela entraîne de violences racistes. Ainsi, la violence et l'insécurité règnent dans de nombreuses villes des EtatsUnis, et pas seulement dans de nombreuses villes du tiers-monde, et la montée d'une 
violence de base raciste et xénophobe, en Allemagne ou en Grande-Bretagne est un phénomène qui a pris en quelques années un essor préoccupant ${ }^{9}$. On peut donc ici faire un pas de plus, et considérer que la mondialisation de l'économie, et ses liens directs avec la fragmentation culturelle et sociale, contribuent à la mondialisation de la violence, avec ses formes éclatées. Par exemple, il est utile de parler de déterritorialisation et de penser globalement, et pas seulement dans leur cadre national, les violences islamistes, de l'Algérie au Pakistan en passant par de nombreux autres pays, de noter l'existence de réseaux, de modes de communication. Mais en même temps, ces violences ne sont pas unifiées dans un projet mondial, bien au contraire, puisqu'elles mettent la plupart du temps en jeu des significations inscrites dans la scène locale ou régionale où elles surgissent.

11 Enfin, l'analyse du système international dans ses changements majeurs, pour être complète, doit être sensible à l'importance et à l'intensité des migrations internationales, qui ne sont pas toutes réductibles à de simples flux d'immigration, et donnent souvent l'image de relations transnationales, diasporiques, ou de " norias " entre deux pays. Les diasporas, notamment, jouent un rôle essentiel dans le domaine de l'économie criminelle et dans le soutien à des mouvements armés - un rôle qui n'est pas nouveau, mais qui s'est accentué et renforcé à travers ce que Aline Angoustures et Valérie Pascal appellent des " phénomènes de mises en réseau "10. classique associe couramment les deux thèmes, ne serait-ce que dans la formulation célèbre de Max Weber, qui écrit, en 1919, que l'Etat " ne se laisse définir sociologiquement que par le moyen spécifique qui lui est propre, ainsi qu'à tout groupement politique, à savoir la violence physique. (...) De nos jours, la relation entre Etat et violence est tout particulièrement intime. (...) Il faut concevoir l'Etat contemporain comme une communauté humaine qui, dans les limites d'un territoire déterminé (...) revendique avec succès pour son propre compte le monopole de la violence physique légitime. Ce qui est en effet le propre de notre époque, c'est qu'elle n'accorde à tous les autres groupements, ou aux individus, le droit de faire appel à la violence que dans la mesure où l'Etat le tolère : celui-ci passe donc pour l'unique source $\mathrm{du}$ " droit " à la violence "11. Définition dont Raymond Aron avait noté l'ambiguïté, soulignant qu'on ne sait pas très bien si le concept proposé ici par Max Weber renvoie à une catégorie abstraite, purement théorique, ou à une catégorie concrète, historique, empiriquement observable ${ }^{12}$.

14 La définition analytique que propose Max Weber s'applique-t-elle aussi bien qu'à son époque aux Etats que nous pouvons observer aujourd'hui ? A bien des égards, les Etats contemporains, ou du moins certains d'entre eux, sont affaiblis. La mondialisation fait qu'ils constituent moins aisément qu'auparavant le cadre territorial, administratif et politique de la vie économique, puisque les flux, les décisions, les marchés, la circulation des hommes, des capitaux, des informations s'opèrent à une échelle planétaire, et d'ailleurs en partie sous des formes illégales qui autorisent à parler d'une mondialisation du crime organisé, notamment à propos de la drogue. De moins en moins capable de contrôler l'économie, l'Etat semble dans de nombreuses situations obligé de reculer face à des activités informelles, au marché noir et au travail clandestin ; la fiscalité devient pour lui difficile à exiger ou justifier, aléatoire, et en même temps que l'économie se privatise, la violence se privatise, moyen de piller l'Etat 
ou de s'attribuer les ressources qu'il devrait contrôler. L'Etat est ainsi débordé du dedans et du dehors, d'autant qu'il se crée ou se renforce des solidarités infra et transétatiques (diasporiques notamment), inscrites ou non sur des territoires précis, dont l'espace de fonctionnement ne correspond plus au sien. De plus, l'Etat, par l'intervention de ses agents, peut pratiquer ou recouvrir une violence illégitime, contraire à son discours officiel, comme en connaissent des pays démocratiques où existent la torture, les abus policiers ou militaires en tous genres, ou bien encore la délégation de l'usage de la force à des acteurs privés qui l'exercent au profit de leurs seuls intérêts ${ }^{13}$. Encore faut-il ajouter ici qu'un débat mérite d'être ouvert, et que, comme le montrent les travaux de Frédéric Ocqueteau sur le secteur marchand de la protection ou sur les politiques de sécurité mises en œuvre dans les centres commerciaux, les effets de la privatisation de l'usage de la force ne sont pas nécessairement à sens unique ${ }^{14}$.

Les Etats éprouvent de plus en plus de peine à assumer leurs fonctions classiques. Le monopole légitime de la violence physique est dès lors comme atomisé et, dans la pratique, la célèbre formule wébérienne semble de moins en moins adaptée aux réalités contemporaines.

16 La fragmentation culturelle contribue elle aussi à cette tendance générale. Elle rend plus délicate à gérer la formule de l'Etat-nation, puisque la nation ne peut pas aussi facilement qu'auparavant réclamer pour elle le monopole ou le primat absolu de l'identité culturelle des personnes réunies au sein de la communauté imaginaire qu'elle constitue, selon l'expression de Benedict Anderson ${ }^{15}$ : d'autres identités s'affirment, demandent à être reconnues dans l'espace public, et les chocs interculturels peuvent prendre l'allure de guerres communautaires. Là où l'Etat est ancien, comme en Europe, il s'affaiblit, là où il est récent, en Afrique, en Asie, " pur produit d'importation " selon une formule lancée par Bertrand Badie et Pierre Birnbaum, il est souvent corrompu, inefficace, délégitimé du fait de ses propres carences, au point qu'on a pu parler de " panne d'Etat " et y voir une source majeure d'insécurité pour la planète : " La première question de sécurité aujourd'hui, affirme Philippe Delmas, ce ne sont pas les ambitions de puissance, c'est la panne des Etats. "16.

Certains parlent sinon de dépérissement de l'Etat, du moins du déclin du modèle qu'il a pu constituer, évoquant par exemple un retour au moyen-âge, un " néomédiévalisme " pour décrire l'affaiblissement des Etats-Nations et rendre compte de l'image qui se préciserait d'une " pluralité de communautés et d'allégeances, hiérarchiques ou enchevêtrées "17 - thème lancé dès les années soixante-dix par Umberto $\mathrm{Eco}^{18}$. D'autres insistent sur un phénomène paradoxal, qui est l'existence, notamment dans des situations où la greffe de l'Etat moderne a abouti à la corruption, à l'inefficacité ou à la délégitimation, de demandes d'insertion dans un ordre international où la protection des territoires viendrait de puissances fonctionnant sur le modèle de l'empire, Ghassan Salamé parle d' "appels d'empire ", celui-ci, explique-t-il, ayant " une relation plus flexible que l'Etat-Nation avec le territoire "19. Retour à l'empire, nouveau moyenâge: les formulations peuvent varier, qui marquent tout à la fois l'idée d'un dépassement de la formule contemporaine de l'Etat, en même temps que d'une réinvention de ce qui fut expérimenté dans le passé.

Ce constat mérite pourtant d'être nuancé. Il n'est d'abord pas certain qu'il faille, partout où l'Etat est récent, dire de sa greffe sur des sociétés auparavant organisées autrement, en royaumes et en empires par exemple, qu'elle est un échec, et bien des 
arguments militent en faveur de l'idée, défendue notamment par Jean-François Bayart, selon laquelle se poursuit, en Afrique ou en Asie, " l'universalisation de quelques-uns des éléments fondamentaux de la civilisation occidentale ", incluant éventuellement l'Etat $^{20}$. Tout n'est pas affaiblissement, décomposition en ce qui concerne l'Etat. Plus généralement, diverses expériences contemporaines suggèrent que le concept analytique d'Etat est loin d'être dépassé historiquement. Ainsi, Olivier Roy, s'intéressant plus particulièrement au Moyen-Orient et à l'Asie centrale, montre que l'Etat y demeure l'horizon indépassable des recompositions politiques, et que les groupes de solidarité infra-étatiques dont il observe les jeux et l'essor, fondés par exemple sur l'ethnicité, ne peuvent pas se passer d'Etat - " les contrebandiers ont besoin de frontières "21. Il est difficile de proposer une représentation unifiée et linéaire de l'évolution actuelle de la formule de l'Etat et de son adéquation aux problèmes politiques de notre époque; et tout en acceptant l'image d'un affaiblissement de nombreux Etats dans leur capacité, qui les définit classiquement, à s'arroger concrètement le monopole légitime de la violence physique et à le faire fonctionner, il faut donc être prudent, et reconnaître qu'à la tendance à la crise de la formule classique de l'Etat, et plus encore de l'Etat-Nation, on peut opposer l'image, moins forte, mais ayant quelque pertinence, de sa pérennité, voire de son avenir.

La pensée contemporaine sur la violence est hantée par l'idée d'un dépérissement/ dépassement de l'Etat. Celui-ci est de moins en moins décrit comme cause ou source ou justification de la violence, comme c'était le cas lorsqu'il s'agissait d'expliquer, dans les années soixante et soixante-dix, les luttes dites de libération, nationale ou sociale, ou de promouvoir des projets révolutionnaires; l'Etat est désormais à nouveau, comme dans l'essentiel de la tradition de la philosophie politique au moins depuis Hobbes, la formule politique qui devrait interdire la violence physique en dehors de son champ d'action et de contrôle, et qui y parviendrait de moins en moins bien. On pourrait certes évoquer de nombreuses expériences où la violence est une réponse à la brutalité de l'Etat, à un pouvoir dictatorial ou à une oppression de type néo-colonial par exemple. On pourrait tout autant indiquer comment, ne serait-ce qu'avec l'expérience palestinienne, elle peut avoir à faire avec le désir de construire un Etat doté de tous les attributs de la souveraineté nationale. Mais la tendance dominante est ailleurs, elle est dans les expériences, beaucoup plus nombreuses, où la violence surgit ou se développe dans les carences de l'Etat.

Un paradoxe ici est que les formes les plus spectaculaires de violence ne correspondent pas nécessairement aux situations de plus grande carence, faiblesse ou absence de l'Etat. Ainsi, comparant la violence urbaine des jeunes dans deux situations présentant bien des points communs, puisqu'il s'agit de quartiers en difficulté dans les banlieues de Lyon et à Milan, Paola Rebughini ${ }^{22}$ constate qu'en région lyonnaise, la violence a souvent revêtu un tour émeutier et spectaculaire, médiatique à la limite, alors qu'à Milan, elle est plus diffuse, plus proche de la délinquance et du crime organisé. Une des sources de cette différence renvoie à l'Etat: plus présent en France, il y est, tout à la fois, l'acteur qui cause la violence (par exemple à partir du racisme policier), et l'acteur que la violence a vocation à interpeller, ce pour des raisons aussi bien instrumentales qu'expressives : une émeute attire l'attention de la presse, et de là, la venue dans le quartier de responsables politiques, qui eux-mêmes éventuellement apporteront des moyens et des ressources nouvelles pour ce quartier. Différemment, en Italie, les espaces concernés vivent en dehors de l'Etat, l'économie clandestine ou illégale y apporte d'importantes ressources, et une violence trop visible, de type émeutière 
précisément, ne pourrait qu'attirer l'attention des médias dont on ne veut pas. La violence assure bien davantage ou bien la survie des uns et des autres (délinquance), ou bien le contrôle de territoires dont la privatisation implique que l'Etat soit le plus possible tenu à distance; et comme l'Etat italien n'a lui-même guère vocation à intervenir, on comprend bien que la violence revête un tour différent de ce que l'on observe en France.

Ajoutons une dernière remarque, très générale, empruntée à Ghassan Salamé : si l'Etat territorial classique s'effrite, alors, la forme de violence qui lui est le plus directement liée, la guerre interétatique, elle aussi prend moins d'importance, au profit d'autres formes, guerres civiles, massacres interethniques par exemple ${ }^{23}$. Ces formes de la violence sont parmi les plus massives et les plus spectaculaires du monde contemporain.

Mutations sociétales

Dans les années cinquante et soixante, la pensée évolutionniste, sous hégémonie nordaméricaine, développait l'idée d'un one best way, selon laquelle les sociétés, dans le monde entier, étaient appelées à s'engager sur les mêmes rails d'une modernisation qui était envisagée en termes économiques - le développement - et en termes politiques - la démocratisation. Dans cette perspective, l'horizon était dessiné par les sociétés industrielles les plus avancées, et donc en premier lieu par les Etats-Unis, et la violence devait elle-même régresser au fur et à mesure du progrès.

Nous savons bien aujourd'hui qu'il existe plusieurs modèles de développement, que les progrès économiques et politiques ne signifient pas nécessairement la régression de la violence, et que les sociétés avancées peuvent fort bien combiner vives difficultés sociales et post-industrialisation. Dans les sociétés occidentales, en effet, la perte de centralité de l'industrie classique va souvent de pair avec des phénomènes de chômage et de précarisation, les rapports d'exploitation dans le travail et la production laissent la place à l'exclusion dans la définition de la question sociale. L'emploi et la croissance se séparent, et dans ce type de changements, le principe de structuration conflictuelle de la vie sociale qu'apportait l'opposition entre le mouvement ouvrier et les maitres du travail se décompose. Dès lors, le syndicalisme et les systèmes de relations professionnelles s'affaiblissent, y compris là où ils avaient une grande vitalité, comme en Allemagne ou dans les pays scandinaves, le couple classique gauche-droite cesse de représenter un conflit social au niveau politique, les attentes politiques s'exacerbent sans trouver de lieu de médiation, les populismes se déploient, de Ross Perot aux EtatsUnis à la Ligue du Nord en Italie en passant par le national-populisme du Front national en France, et la crise sociale se combine avec la question des identités culturelles, nationales, ethniques, religieuses pour alimenter des violences qui confortent les unes les tendances à l'éclatement des sociétés nationales, les autres les appels réactifs à l'ordre qui se défait.

Des transformations comparables affectent les sociétés des anciens pays de l'Est, pour qui la fin de l'expérience soviétique est aussi la déstructuration d'un modèle centré sur le lieu de travail qui apportait à chacun des garanties dont beaucoup étaient gérées depuis l'entreprise - emploi, logement, accès à la santé, au loisir, consommation de base, etc. Mais ne déduisons pas de ces constats l'idée d'une violence sociale ou politique directement liée à l'épuisement des rapports sociaux propres à l'industrie classique. S'il y a certainement un lien entre la violence et ces changements sociaux, ce lien n'est pas automatique et immédiat, la violence doit être pensée en faisant 
intervenir des médiations. Elle ne surgit pas directement de la mobilité sociale descendante, ou de la crise ; ainsi, les émeutes des quartiers de relégation de France ou d'Angleterre, celles des grandes métropoles américaines, surviennent à l'occasion d'excès policiers ou de décisions de justice inadéquates, bien plus qu'elles ne protestent contre le chômage; la rage et la haine des jeunes s'expriment certes sur fond de difficultés sociales, mais elles correspondent d'abord à des sentiments puissants d'injustice et de non-reconnaissance, de discrimination culturelle et raciale. Le chômage et la pauvreté, y compris lorsqu'ils traduisent une chute sociale brutale, comme dans les pays de l'ancien empire soviétique, ne se soldent guère immédiatement ou directement en violences sociales - ce que l'on savait depuis l'étude classique de Lazarsfeld sur les chômeurs de Marienthal ${ }^{24}$-, mais plutôt nourrissent des frustrations qui transitent éventuellement par un nationalisme exacerbé, ou un appel au retour des communistes. Et s'ils alimentent des violences collectives, celles-ci sont plus racistes et antisémites, plus ou moins liées à des références nationalistes, que proprement sociales.

\section{L'individualisme contemporain}

L'individualisme, tel qu'il se manifeste avec une force croissante dans le monde contemporain, présente deux faces, complémentaires et éventuellement opposées. D'une part, l'individu moderne veut participer à la modernité, à ce qu'elle offre, qu'elle promet, qu'elle donne à voir à travers ses médias et les sollicitations d'une consommation de masse dont le spectacle est désormais mondialisé. Il entend consommer, continuer à consommer s'il l'a déjà fait, commencer à le faire s'il n'a pas encore pu. Et d'autre part, l'individu veut être reconnu comme sujet, construire sa propre existence, ne pas être totalement dépendant de rôles et de normes, pouvoir s'en distancier sans être pour autant obligé de le faire. Il entend par exemple effectuer des choix qui l'autorisent à procéder d'une identité collective, sans lui être totalement subordonné, se produire, et pas seulement se reproduire.

Ces deux faces de l'individualisme ne sont pas une nouveauté, et d'une certaine façon, on en trouve l'évocation chez Emile Durkheim lorsqu'il distingue l'individu, qui relève du mode profane, et la personne, qui relève à ses yeux du sacré25. Elles entretiennent chacune, aujourd'hui, un rapport assez fort à la violence, même collective. L'acteur de bien des violences instrumentales, par exemple, s'engage à des fins économiques, il veut de l'argent pour consommer, acheter, pour lui-même et pour les siens éventuellement. Assez différemment, la violence peut revêtir un tour extrême, sans limites, en relation avec un désir, frustré, d'accéder aux fruits de la modernité, et sans qu'il s'agisse de l'utiliser comme ressource pour parvenir à des fins. Ce qui la rapproche de conduites informées par la rage de ne pas être reconnu, par le sentiment d'une injustice vécue, par l'interdiction qui est faite à l'individu de devenir sujet, et qui peuvent prendre différentes formes : explosion émeutière, mais aussi ludique, ou, pour parler comme David Le Breton ${ }^{26}$, informée par les " passions du risque " qui peuvent alors, à la limite, devenir ordaliques ou autodestructrices, retournement contre soi de l'impossibilité créée par le système ou la situation à être acteur de son existence. La violence est ici ou bien quête et production de sens, effort pour produire soi-même ce qui était auparavant dispensé par la culture ou les institutions, projection de soi-même jusqu'à la mort éventuelle, ou bien appel à la subjectivité impossible ou malheureuse, expression d'un refus de la personne à continuer une existence où elle se sent niée. Dans cette dernière perspective, le racisme subi, en particulier, en expansion dans de 
nombreuses sociétés, est une expérience qui est largement vécue par ses victimes comme une profonde négation de leur individualité, ce qui peut se transformer en rage et de là en violence par exemple émeutière. Tout ceci n'est certes pas nouveau ; mais les progrès de la mondialisation rendent bien plus aigus que par le passé tout ce qui renvoie à l'individualisme, ainsi qu'aux fragilités personnelles qui vont de pair, surtout lorsqu'il s'agit de combiner les deux registres, de l'efficacité instrumentale, stratégique, et de la construction d'une subjectivité autonome ${ }^{27}$ : personne désormais, en effet, n'ignore ce que le monde moderne peut offrir ou promettre, aussi bien comme possibilités de consommation qu'en matière de production de soi-même, mais il est bien difficile d'être à la fois consommateur et producteur de son existence, efficace et rationnel d'un côté, autonome et distancié par rapport aux normes d'un autre côté. La violence trouve ici un triple faisceau de conditions favorables: soit que la pure rationalité stratégique commande d'en faire, plus ou moins cyniquement, une ressource; soit que le souci de s'identifier à une identité collective aboutisse au fanatisme ou à un sectarisme guerrier; soit enfin, dans des processus de fusion du sens, où la double impossibilité de fonctionner comme consommateur et comme producteur de son existence trouve à se résoudre dans une invention de sens imaginaire d'autant plus violente qu'elle ne trouve pas hic et nunc les moyens concrets de se transcrire en pratiques.

Ajoutons que l'individualisme exerce ses effets non seulement sur les significations, mais aussi sur les formes que peut revêtir la violence contemporaine, ce qui semble pouvoir constituer une des leçons que tire Jean-Paul Grémy d'un ensemble d'études portant sur les violences urbaines en France, lorsqu'il note que les bandes de jeunes, ou leurs regroupements à l'occasion d'émeutes dans les quartiers de relégation, procèdent d'un individualisme qui " rend particulièrement difficile les négociations en vue de l'arrêt des violences "28.

La séparation entre niveaux ou registres peut ainsi constituer un instrument utile d'analyse. Ajoutons aussi qu'elle ne doit pas pour autant conduire à un éclatement intellectuel, dans lequel les chercheurs se spécialiseraient sur l'un ou l'autre de ces niveaux ou registres. Au contraire, la réflexion sur la violence a tout à gagner non seulement à prendre en considération des sous-ensembles clairement délimités - le système international, l'Etat, etc., mais aussi à penser leur complémentarité, leur articulation, ou au contraire leur dissociation. Peut-être même les remarques qui précèdent appellent-elles une suggestion : ne vaudrait-il pas mieux, contrairement à la tradition intellectuelle des sciences politiques, partir du bas, de l'individualisme ou des transformations qui affectent les rapports sociaux, et remonter pour l'analyse jusqu'au niveau international, dont les changements, sous leurs formes réelles ou plus ou moins mythiques (la globalisation) s'alimentent du travail des acteurs et des sociétés sur ellesmêmes, au moins autant qu'ils les informent?

Doutes et incertitudes

Violence objective, violence subjective

Présentés certes rapidement, les changements qui affectent donc les quatre niveaux que nous avons distingués appellent une sociologie capable de les étudier de manière analytique, séparément, mais aussi de les aborder globalement. Il n'existe pas une sociologie intégrée de la violence, qui proposerait une théorie unifiée satisfaisante, permettant d'embrasser simultanément les niveaux de la personnalité et de l'individu, ceux de la société, de l'Etat et du système des relations internationales; mais cela 
n'interdit pas de s'efforcer de ne pas les séparer dans la réflexion, et notamment de faire l'hypothèse qu'une des sources fondamentales de la violence contemporaine réside précisément dans leur tendance à la dissociation. Par exemple, il est tentant d'analyser d'un côté la personnalité de jeunes " sicaires ", tueurs à gages qui, en Colombie, semblent conduits par un désir d'argent et de consommation puissant et sans barrières morales, et d'un autre côté, de traiter de l'économie planétaire de la drogue ou des relations entre les Etats-Unis et la Colombie; mais il est beaucoup plus utile de montrer comment la violence instrumentale du jeune tueur à gages de Médellin oscille entre la politique et la criminalité, et s'inscrit dans tout un tissu de relations sociales et politiques, au niveau du quartier, de la ville, du pays, du continent, qui ne se limitent pas à l'image de deux univers disjoints, international (la drogue et son économie), et personnel ou psychologique (l'absence de normes intériorisées qui rend possible le fait d'être un tueur) ${ }^{29}$.

Plus on entre dans ce type d'analyse, plus il devient utile de se demander quelle est la part de la violence subjective, et celle de la violence objective. Entre l'acteur, réduit à la limite à sa personnalité ou sa subjectivité, et le système, référé à des processus planétaires dont la notion de mondialisation est devenue le symbole, la violence vient emplir le vide qu'ont laissé des acteurs et des rapports sociaux et politiques défaillants. Mais un vide réel, ou un vide dans les représentations? La tâche d'une sociologie de la violence est de montrer les médiations absentes, les systèmes de relations dont le manque ou l'affaiblissement créent l'espace de la violence: si ces médiations, si ces systèmes de relations sont plus cachés, incompris ou ignorés que réellement carents ou absents, parce que la société concernée, ses élites politiques, ses intellectuels, son opinion se refusent à les connaître et à en débattre, alors la violence doit être analysée avant tout comme une représentation, comme la subjectivité de groupes, voire d'une société tout entière incapables de se comprendre et de comprendre leur environnement; s'ils sont tangibles, s'il est empiriquement établi qu'il y a déficit d'acteurs et de médiations par des systèmes de relations, la violence est certainement une forte réalité objective. La sociologie doit ici faire la part des choses, montrer comment la violence contemporaine se renouvelle aussi bien dans ses perceptions subjectives que dans ses réalités historiques.

C'est pourquoi l'hypothèse d'un changement de paradigme de la violence renvoie aussi à un problème classique de la sociologie de la connaissance : la façon dont nous parlons de la violence et de ses changements contemporains, y compris dans le discours savant, est-elle simplement commandée par l'état du phénomène et par ses transformations objectives, ou par des changements les plus divers, qui se jouent aux différents niveaux qui ont été distingués ici, mais de façon relativement autonome par rapport aux faits de violence, influant sur nos perceptions et façonnant des représentations qui ne correspondent que plus ou moins artificiellement aux expressions concrètes du phénomène? D'une expérience concrète à une autre, la réponse ne peut que varier : l'essentiel ici est d'insister sur la nécessité qu'il y a à poser la question.

Désarticulation

Si la violence a à voir avec des changements qui affectent non seulement chacun des quatre niveaux retenus pour l'analyse, mais aussi avec leur ensemble, c'est avant tout parce que ces niveaux semblent se désarticuler à partir du moment où on les considère dans leurs expressions concrètes, et pas seulement comme des catégories analytiques. Le plus décisif renvoie ici à la crise de l'Etat-Nation, affaibli dans son rôle de cadre ou 
d'espace principal, territorial, politique, administratif et intellectuel de la vie collective. Ainsi, il y a hiatus entre l'échelle des problèmes, planétaires, que posent l'économie et l'écologie, et les outils institutionnels, essentiellement étatiques, dont on dispose pour les traiter. A l'intérieur même de chaque pays, il devient souvent compliqué d'établir une forte correspondance entre diverses politiques, de rendre cohérentes, par exemple, la politique économique et commerciale et la politique étrangère. Ou bien encore, il est difficile de concilier la diplomatie, voire la participation d'un Etat à des institutions internationales, et la sensibilité individuelle croissante au thème des droits de l'homme, qui peut être une incitation à des interventions autres qu'étatiques, liées au droit d'ingérence. La désarticulation est la plus spectaculaire là où, société, Etat et culture formaient un ensemble relativement intégré, dans ce que, à la suite d'Alain Touraine, on peut appeler des sociétés nationales ${ }^{30}$, ou rapports sociaux propres à l'ère industrielle, institutions assurant égalité individuelle et solidarité collective, et identité nationale formaient un système à forte cohérence, autosuffisant ou presque, qui se déstructure aujourd'hui sous l'effet du néolibéralisme et de la mondialisation.

La violence, de ce point de vue, peut résulter de l'effort de certains acteurs pour maintenir de façon de plus en plus artificielle ou volontariste ce qui se défait; elle s'exprime, éventuellement, par des agressions à l'encontre de ceux qui sont accusés ou soupçonnés d'incarner et de mettre en oeuvre la désintégration socio-politique de la société nationale, et d'être le vecteur de l'hétérogénéité culturelle qui menacent. Elle vise alors, en priorité les immigrés, et plus largement, les groupes humains qui peuvent être le plus facilement racisés. Le racisme en Europe, y compris dans ses expressions les plus actives, les plus destructrices et meurtrières, a beaucoup à voir avec le refus réactif de la décomposition des sociétés nationales.

\section{Un monde sans repères?}

Pour penser les formes et l'importance de la violence, n'est-il pas utile de disposer de repères permettant de distinguer, dans l'espace, des zones relativement homogènes ? Trois réponses principales, éventuellement combinées, peuvent tenter d'apporter une réponse constructive à cette interrogation : politiques (ou géopolitiques), économiques et culturelles.

Dans les années de la guerre froide, la violence pouvait être appréhendée à partir d'un découpage géopolitique tenant compte de la bipolarité du monde. Elle avait d'autant moins de chances sinon de surgir, du moins de s'étendre et de revêtir un tour politique, qu'elle risquait de mettre en cause les équilibres fondamentaux entre l'Est et l'Ouest. L'Europe était au coeur de ces équilibres, ce qui ne pouvait pas empêcher de graves violences, mais leur interdisait tout débouché politique ou géopolitique majeur. Il était alors possible de distinguer trois " mondes " ou sous-ensembles de pays : l'Occident, incluant l'Amérique du Nord et l'Europe de l'Ouest, le monde communiste, et enfin les pays en voie de développement, le tiers-monde, avec ses conflits intérieurs pouvant être très violents, et des pouvoirs d'Etat plutôt faibles et instables. Avec la chute du mur de Berlin, la difficulté à penser le monde à partir de distinctions politiques ou géopolitiques est devenue si considérable qu'on a pu parler de fin de l'histoire ${ }^{31}$.

Une autre distinction, économique, oppose le Nord, riche, et peu susceptible d'être affecté par de graves violences, et le Sud, exclu des réseaux et des flux principaux de la mondialisation, et propice aux pires violences civiles, ethniques ou autres. Mais l'exclusion exerce des ravages si profonds au sein même des sociétés les plus avancées, 
et les différences internes aux pays du Sud sont si considérables qu'il est difficile de penser la violence à partir de ce clivage.

Enfin, un troisième clivage, culturel, a été récemment proposé pour distinguer des civilisations, avec l'idée que la violence allait se développer, dans le monde contemporain, entre les grandes civilisations, là où elles s'entrechoquent - thèse de Samuel Huntington dont nous avons vu qu'elle revient à sous-estimer les tensions et les différences culturelles produites et reproduites au coeur même de chaque civilisation ${ }^{32}$.

Aujourd'hui, le tiers-monde est au coeur des sociétés les plus développées, le communisme s'est effondré et avec lui l'idée d'une opposition Est-Ouest, de nouvelles puissances s'ébauchent, comme dans le Pacifique Sud, la violence a surgi, particulièrement meurtrière au Nord, et notamment en ex-Yougoslavie; elle combine partout des affirmations culturelles et des demandes socio-économiques, tout se brouille, au point que Ghassan Salamé parle de disparition des " quatre points cardinaux ". Ajoutons que les meilleurs analystes expliquent que la notion de tiersmonde, si utilisée dans le passé, était une commodité de langage déjà bien artificielle à l'époque, un concept amalgamant des réalités hétérogènes.

Pour penser de manière différenciée le surgissement et le développement de la violence dans l'espace, il n'y a donc plus de principe géopolitique fort, les distinctions économiques sont insuffisantes, et la thèse du choc des civilisations s'avère inappropriée. Dans un monde à la fois fragmenté et globalisé, les probabilités de graves violences localisées sont grandes partout, et en même temps, les problèmes même les plus circonscrits ont bien plus que par le passé toutes les chances d'être déplacés, exportés, prolongés en dehors de leur espace initial ou naturel. La violence lorsqu'elle est liée à une cause nationale, peut revêtir un tour diasporique, et introduire de la complexité là où on ne l'attendait pas. Par exemple, la question kurde est devenue d'une importance non négligeable dans la vie interne et diplomatique de l'Allemagne, où des violences ont pu survenir en raison de la présence dans ce pays d'une forte population immigrée originaire de Turquie. De même, ce n'est pas parce que l'immigration d'origine algérienne en France s'efforce de s'intégrer massivement, dans l'ensemble, qu'il n'existe pas en son sein de sensibilité diasporique, voire des réseaux dont certains fonctionnent à l'échelle internationale et peuvent être liés à des activités de violence armée en Algérie. Ou bien encore, il est possible d'établir des liens entre la crise de tel ou tel Etat central, et celle de tel ou tel Etat du tiers-monde, ce qui apparait de façon spectaculaire si l'on considère le caractère non seulement piteux, mais aussi scandaleux, de certaines interventions politiques ou militaires, du type de celles qu'a pu recevoir la Somalie. " Les interventions des pays du " Centre " (sous forme plus ou moins onusienne) écrit Alain Joxe, ne sont pas de pures tentatives de remise en ordre à partir des sociétés " ordonnées ", mais des actions elles-mêmes désordonnées et désordonnantes, reflétant au départ la crise " centrale " des représentations et des acteurs de l'échelle légitime du monopole de la violence, les Etats. Il y a de la complexité et de la confusion aux deux bouts de l'expédition. " ${ }^{33}$.

Ce qui nous permet de préciser l'idée d'un nouveau paradigme de la violence : celle-ci doit effectivement être abordée, désormais, avec des concepts qui ne peuvent plus être ceux d'un monde bipolaire, ni ceux d'un monde où l'économie permettait de penser directement les rapports internationaux en termes de domination et d'exploitation, ou de modernisation plus ou moins avancée. Elle doit être appréhendée dans ce qui est une de ses nouveautés radicales: le fait d'être à la fois globalisée, puisque référable à des 
phénomènes planétaires, et localisée; générale, et, pour utiliser le terme de Hans Magnus Enzensberger ${ }^{34}$, moléculaire ; elle-même mondialisée, et fragmentée ou éclatée. Elle n'est pas forcément différente selon que l'on considère le centre ou la périphérie, notions qu'elle vient contribuer à affaiblir; et par exemple il y a bien des similitudes entre les jeunes acteurs des mouvements islamistes au Moyen Orient, et les jeunes enragés des banlieues françaises, qu'ils soient ou non musulmans. Ce caractère singulier de la violence contemporaine nous oblige encore plus à réfléchir en allant d'un extrême, socio-historique, à l'autre, centré sur la personne. La violence nous interroge, non pas parce que, plus qu'à d'autres moments, nous irions vers le chaos généralisé, ou même parce que nous serions immergés dans l'incertitude accrue des lendemains de guerre froide, mais parce que nous devons apprendre à la concevoir autrement, avec la plus vive conscience d'une nouvelle donne historique et politique. Nous devons nous méfier des affirmations trop pressées qui ne veulent voir que du néo ou du retour, là où les phénomènes considérés ou bien sont réellement si nouveaux qu'ils appellent un renouvellement total de nos catégories, ou bien sont plus anciens et plus complexes que ne le suggèrent ces termes qui manquent vite de nuance, tout comme nous devons résister à l'idée d'évolutions linéaires ou de conséquences unidimensionnelles de tel ou tel phénomène - la mondialisation de l'économie, par exemple, n'exerce pas d'effets uniformes, mais bien davantage accentue de façon ambivalente certaines tendances, comme celles qui, simultanément, assurent l'internationalisation de la consommation de masse ou des produits télévisuels, et la fragmentation culturelle; et ce n'est pas parce que la France a tardé considérablement à la découvrir qu'il s'agit d'un phénomène récent.

Revenir à la partie précédente

\section{NOTES}

1. Pierre Hassner, op. cit., p. 11.

2. Michel Wieviorka, Sociétés et terrorisme, op. cit., chap. 2 de la Première partie.

3. . Pierre Hassner, op. cit., p. 55.

4. Philippe Delmas, Le bel avenir de la guerre, Paris, Gallimard, 1995.

5. Cf. François Jean et Jean-Christophe Rufin (dir.), Economie des guerres civiles, Paris, Hachette, coll. Pluriel, 1996

6. Cf. par exemple Richard O'Brien, The End of Geography : Global Financial Integration, Londres, Piner, RIIA, 1992.

7. Elie Cohen, La tentation hexagonale. La souveraineté à l'épreuve de la mondialisation, Paris, Fayard, 1996.

8. Benjamin Barber, "Jihad Vs. Mc World ", in The Atlantic Monthly, march 1992, p. 53-65 ; cf. aussi en français, du même auteur, le livre qui porte ce titre, Djihad versus McWorld, Paris, Desclée de Brouwer, 1996.

9. Cf. les textes d'Angelina Peralva sur l'expérience allemande, et de Kristin Couper et Danilo Martuccelli sur l'expérience britannique, dans Michel Wieviorka (dir.), Racisme et xénophobie en Europe. Une comparaison internationale, Paris, La Découverte, 1994. 
10. Aline Angoustures et Valérie Pascal, " Diasporas et financement des conflits ", dans Economie des guerres civiles, op. cit., p. 495-542.

11. Max Weber, Le savant et le politique, Paris, Plon, "10-18", 1963 (1ère éd. 1919), p. $124-125$.

12. "Max Weber n'a pas choisi entre des concepts purement analytiques et des concepts semi-historiques ", écrit Raymond Aron dans Les étapes de la pensée sociologique, Paris, Gallimard, "Tel", 1967, p. 559, cité par Pierre Bouretz, Les promesses du monde. Philosophie de Max Weber, Paris, Gallimard, 1996, p. 263. 13. Par exemple, cf. Paulo Sergio Pinheiro, "Institutions and Impunity : Violence, Crime and Police System in New Democratic Countries (the Brazilian Experience in the Context of Latin American Countries ", paper presented at the international seminar Strategies of Police Intervention in the Modern State, São Paulo, sept. 1996.

14. Frédéric Ocqueteau, " Etat, compagnies d'assurances et marché de la protection des biens ", dans Déviance et Société, vol. 19, n², p. 151-158 ; " Les centres commerciaux, cibles d'incivilités et promoteurs de sécurité ", dans Déviance et Société, vol. 17, n 3, p. 235-260 ; " L'Etat face au commerce de la sécurité ", dans L'Année sociologique, n40, 1990, p. 97-124.

15. Benedict Anderson, Imagined Communities. Reflections on the Origin and Spread of Nationalism, Londres, Verso, 1983.

16. Philippe Delmas, Le bel avenir de la guerre, Paris, Gallimard, 1995, p. 9.

17. Pierre Hassner, op. cit., p. 56.

18. Umberto Eco, " Verso un nuovo Medievo ", in Dalla periferia dell'imperio, cité par Pierre Hassner, op. cit., p. 56.

19. Ghassan Salamé, Appels d'empire. Ingérences et résistances à l'âge de la mondialisation, Paris, Fayard, 1996, p. 21

20. Jean-François Bayart, " L'historicité de l'Etat importé ", dans Les Cahiers du CERI, n 15,1996, p. 6.

21. Olivier Roy, " Groupes de solidarité au Moyen-Orient et en Asie centrale ", dans Les Cahiers du CERI, n 16, 1996.

22. Paola Rebughini, Violence juvénile dans les quartiers défavorisés. Enquête comparative (Lyon-Milan), Mémoire de DEA, Paris, EHESS, 1994.

23. " Tout comme l'émergence de l'Etat territorial, il y a deux ou trois siècles, était allée de pair avec la guerre interétatique, l'expliquant et s'en nourrissant, l'effritement de l'Etat territorial et la prolifération des guerres civiles sont en réalité deux processus qui se renforcent mutuellement, l'un éclairant et favorisant l'autre ", Ghassan Salamé, op. cit., p. 95.

24. Paul Lazarsfeld et al., Les chômeurs de Marienthal, Paris, Minuit, 1981.

25. Emile Durkheim, Les formes élémentaires de la vie religieuse, Paris, PUF, 1968.

26. David Le Breton, Passions du risque, Paris, Métailié, 1991.

27. Cf. Alain Ehrenberg, L'individu incertain, Paris, Calmann-Lévy, 1995.

28. Jean-Paul Grémy, Les violences urbaines, Paris, IHESI, 1996, p. 11. Notons ici qu'il n'est pas certain que l'individualisme dont parle Jean-Paul Grémy corresponde à la définition que nous venons de proposer.

29. Cf. par exemple Carlos Miguel Ortiz Sarmiento, " El sicariato en Medellin : entre la violencia politica y el crimen organizado ", dans Analisis Politica, n 14, sept.-déc. 1991, p. 60-73.

30. Cf. Alain Touraine, Critique de la modernité, Paris, Fayard, 1992. 
31. Francis Fukuyama, La fin de l'histoire et le dernier homme, Paris, Flammarion, 1992.:

32. Pour Susan George, Samuel Huntington et Francis Fukuyama sont portés par le même courant idéologique, ce que confirmerait le fait que tous deux ont bénéficié de fonds Olin, destinés à " renforcer les institutions économiques, politiques et culturelles sur lesquelles est basée l'entreprise privée " (Susan George, dans Le Monde Diplomatique, août 1996).

33. Alain Joxe, " Nouveau paradigme stratégique : la révolution dans les affaires militaires ou la guerre de l'information ", dans Cahiers d'Etudes Stratégiques, op. cit. , p. 85 .

34. Hans Magnus Enzensberger, La Grande Migration, suivie de Vues sur la guerre civile, Paris, Gallimard, 1995.

INDEX

Mots-clés : sociologie des conflits (polémologie), violence, sociologie 